\title{
Using learning analytics to develop early- warning system for at-risk students
}

\author{
Gökhan Akçapınar * (iD, Arif Altun and Petek Aşkar
}

\author{
* Correspondence: gokhana@ \\ hacettepe.edu.tr \\ Department of Computer Education \\ and Instructional Technology, \\ Hacettepe University, Ankara 06800, \\ Turkey
}

\begin{abstract}
In the current study interaction data of students in an online learning setting was used to research whether the academic performance of students at the end of term could be predicted in the earlier weeks. The study was carried out with 76 secondyear university students registered in a Computer Hardware course. The study aimed to answer two principle questions: which algorithms and features best predict the end of term academic performance of students by comparing different classification algorithms and pre-processing techniques and whether or not academic performance can be predicted in the earlier weeks using these features and the selected algorithm. The results of the study indicated that the kNN algorithm accurately predicted unsuccessful students at the end of term with a rate of $89 \%$. When findings were examined regarding the analysis of data obtained in weeks 3, 6, 9,12 , and 14 to predict whether the end-of-term academic performance of students could be predicted in the earlier weeks, it was observed that students who were unsuccessful at the end of term could be predicted with a rate of $74 \%$ in as short as 3 weeks' time. The findings obtained from this study are important for the determination of features for early warning systems that can be developed for online learning systems and as indicators of student success. At the same time, it will aid researchers in the selection of algorithms and pre-processing techniques in the analysis of educational data.
\end{abstract}

Keywords: Academic performance prediction, Early-warning systems, At-risk students, Online learning, Educational data mining, Learning analytics

\section{Introduction}

The prediction of academic performance is one of the first and most popular subjects in the fields of learning analytics and educational data mining (Chatti, Dyckhoff, Schroeder, \& Thüs, 2012; Peña-Ayala, 2014; Romero, Olmo, \& Ventura, 2013). Academic performance can be defined as the score obtained by students from an evaluation made at the end of a learning activity. This score could belong to a short-term learning activity (i.e. a learning object), a lesson, an academic term, or to a complete educational process, i.e., GPA (Peña-Ayala, 2014). Studies have indicated that the learning traces left by students in online learning settings (participation in discussions, spending time on the learning material etc.) are significant in predicting their academic performance.

Lopez, Luna, Romero, and Ventura (2012) analysed the relationship between students' academic performance and their interactions in the discussion forum in the

(c) The Author(s). 2019 Open Access This article is distributed under the terms of the Creative Commons Attribution 4.0 International License (http://creativecommons.org/licenses/by/4.0/), which permits unrestricted use, distribution, and reproduction in any medium, provided you give appropriate credit to the original author(s) and the source, provide a link to the Creative Commons license, and indicate if changes were made. 
Moodle learning management system. The researchers identified eight features of student participation in the discussion forum. By using these features, they attempted to predict student success as pass or fail. Furthermore, they compared the performance of various classification algorithms based on the classification accuracy. According to their findings, the Naive Bayes algorithm reached a classification accuracy of $89.4 \%$, carried out via six features that was determined as a result of the feature selection process. The study findings also revealed that the participation of students in the discussion forum in the Moodle setting could be a reliable feature in the prediction of students' academic performance.

In another study carried out in the Moodle setting by Romero, Ventura, Hervás, and Gonzales (2008), the performance of a group of classification algorithms was compared in order to observe which one would predict the academic performance the most accurate. The researchers selected nine different features that had reflected students' use of Moodle in addition to their participation data in the discussion forum. Students' academic performance was coded as pass, fail, good, or excellent. Upon examination of the research findings, it was observed that the classification accuracy of algorithms varied in the range of 60 to $70 \%$. The research results indicated that students' low usage of online learning system could be one of the reasons behind the low accuracies.

Osmanbegović and Suljić (2012) established a classification model in order to predict the success of students in university using data relating to their demographics and preuniversity success. They used students' final score in the course of "Management Informatics" as the feature for success. The researchers reported that the Naive Bayes algorithm correctly classified students with a rate of $76.65 \%$ using the given set of data. In another study that aimed to predict student performance using interaction data obtained from the learning management system, Macfadyen and Dawson (2010) identified fifteen features that had indicated a statistically meaningful relationship with the students' academic performance. They used the logistic regression method in order to predict the academic performance of students using these features. The researchers stated that the established classification model accurately classified unsuccessful students with a rate of $81 \%$. The number of messages sent to the discussion forum, number of sent emails, and the number of completed assignments were identified as important features in predicting end of term grades. This study further indicated that pedagogically meaningful information could be produced from data on student interactions obtained from learning management systems.

The majority of studies predicting academic performance were carried out using data collected at the end of term. The results obtained from those studies were significant in terms of their determination of features which might lead to a failure; however, they did not provide excessive information to prevent failure. On the other hand, online learning settings constantly collect student interaction data. The prediction models that could be established with the analysis of this data in the earlier stages would be effective in the prevention of possible failures and help instructional designers design and develop pedagogical intervention systems (Costa, Fonseca, Santana, de Araújo, \& Rego, 2017), which are also referred to as early warning systems. In the following section, early warning systems will be explained in detail from educational perspective. 


\section{Educational early warning systems}

Early warning systems can be regarded as the next step in the prediction of academic performance. The aim here is the prediction of academic performance at an earlier stage using features that are derived from online learning settings. As such, teachers may be able to identify students under risk of academic failure and offer assistance in order to help these students make improvements (Campbell, DeBlois, \& Oblinger, 2007). In other words, the performance exhibited by students in lessons could be predicted in advance and possible failures can be prevented by timely interventions (Johnson, Smith, Willis, Levine, \& Haywood, 2006).

Although educational early warning systems have received more attention in recent years, the Signal Project implemented at Purdue University (Indiana, USA) has been cited as a key example of a successful early warning system in the literature (K. E. Arnold, 2010). Instructors are provided a Signal plug-in to be used in their online environments. This plug-in allocates a risk indicator for each student by analysing their interactions (e.g. whether students have read online course materials assigned by the professor, done practice assignments, attended tutorial sessions after class or participated in online class discussions etc.) through the aid of prediction algorithms. The risk indicator structured as a traffic light provides feedback to the student regarding their lesson performance in the form of red (indicating a high probability of failure), yellow (medium probability of failure), and green (high probability of success) lights. A notable improvement in student success was observed in lessons which utilized the Signal system in the pilot scheme that was implemented in the university over a 2 -year period. In the first week of a lesson attended by 220 students, 45 students were placed in the red (high risk) and 32 in the yellow (medium risk) categories. In the progressive weeks, $55 \%$ of students in the red group moved up to the yellow group ( $\mathrm{C}$ level) and $24.4 \%$ to the green group (A or B level), while 10.6\% remained in the red group. It was observed that $31 \%$ of students starting in the yellow group remained in in the same group while $69 \%$ moved up to the green group.

$\mathrm{Hu}$, Lo, and Shih (2014) analysed students' interaction data in an online lesson via data mining techniques in order to develop an early warning system that would predict the learning performance exhibited by students in a course. They set various classification models that would predict students' pass/fail status in lessons using the data from weeks 4,8 , and 13 . The researchers concluded that their early warning system successfully predicted students' learning performances. The experimental data presented by the researchers indicated that the Classification and Regression Tree (CART) algorithm, supplemented by AdaBoost achieved over 90\% accuracy in all datasets. On the other hand, the time dependent features derived from the learning management system had been stated as significant features in predicting student performance.

Costa et al. (2017) benefited from machine learning techniques in order to reduce the high level of failure in the subject of Introductory Programming. The researchers investigated the effectiveness of four different prediction algorithms in the early identification of students with a high probability of failure. The researchers stated that the model established with the Support Vector Machine (SVM) algorithm was effective in the early prediction of unsuccessful students with a rate of $83 \%$. At the same time, this study indicated that the pre-processing techniques were effective in increasing the performance of prediction algorithms. 
Previous studies showed that early prediction of academic failure is possible, however, transferability and generalizability of prediction models into the different courses are still limited. One of the major reasons behind this is because of different instructional conditions used in the course also affects the performance of prediction models (Gašević, Dawson, Rogers, \& Gasevic, 2016). Therefore, it is important to develop and validate prediction models for different courses. In this study, we aimed at developing an early warning system for a blended course and compared not only different algorithms but also different pre-processing techniques effects on algorithms' prediction performances. Feature sets used in the study are also different from existing studies. The results obtained here will be helpful in designing early warning system for the online learning environments and exploring new features to be used in these systems.

The remainder of this paper is structured as follows: the second section provides information about the aims of the study and the research questions to be addressed. The third section explains the research methods, data pre-processing, data analysis techniques, and the online learning setting where the data is collected. The fourth section provides information on the findings. In the conclusion, the findings are discussed in the light of other research findings and suggestions are made for future research.

\section{Aims of the research and research questions}

This study aims to predict the academic performance of students from "Computer Hardware" course, using interaction data in a blended learning setting. In a two-stage research process, the first stage aimed to use the whole data set to determine the algorithm, pre-processing techniques, and features that would best predict the students' end-of-term performances. The second stage investigated whether or not the students' academic performance could be predicted in the earlier weeks using these features and analyzed through the selected algorithm. The research questions related to these stages are listed below:

1. When classification models formed using all data (14 week) are compared with respect to performance metrics, which classification algorithm/algorithms and preprocessing techniques are best in predicting unsuccessful students?

1.1. What is the impact of different data transformation techniques used in the preprocessing stage on classification performance?

1.2. What is the impact of different "feature selection" techniques used in the preprocessing stage on classification performance?

1.3. How is the performance of classification models established using fewer number of features in comparison to other classification models formed using all features?

1.4. Which features carry greater importance in the prediction of students' performance?

2. What is the accuracy of classification models established through data obtained from weeks $3,6,9,12$, and 14 using the selected algorithm and pre-processing techniques within the scope of the first research question in predicting students end of term academic performance? 


\section{Method}

\section{Participants and the data collection process}

The study was conducted at a state university in Turkey with 76 students (41 women and 35 men; age range: 21 to 23 years) registered in the Computer Hardware course in the Department of Computer Education and Instructional Technology. Students participated in online learning activities for a period of 14 weeks and in face-to-face lessons in a blended learning environment. The online learning environment developed by the first author was used for online activities (Fig. 1). The online environment enabled the researchers collect detailed data regarding the online learning processes of students, who used the online learning environment to follow the lesson's resources (Fig. 1-a), participate in discussions relating to the lesson (Fig. 1-b), and write down reflections (posts) regarding the concepts learned in the lessons (Fig. 1-c). This learning environment also has features like notification page where students can track all the activities conducted by their classmates, and an announcement page where students can follow course updates.

The students were registered on the system in the beginning of the semester by researchers and were provided usernames and passwords to log into the system. The students were instructed in how to use the online learning system in the first week of the course. It was clearly explained to them that their reflective writings regarding the concepts they learned or will learn, their participation in online discussions such as reading the messages written by other classmates, and their reflections/evaluations were expected. Students were informed that the reflections written down in the online learning

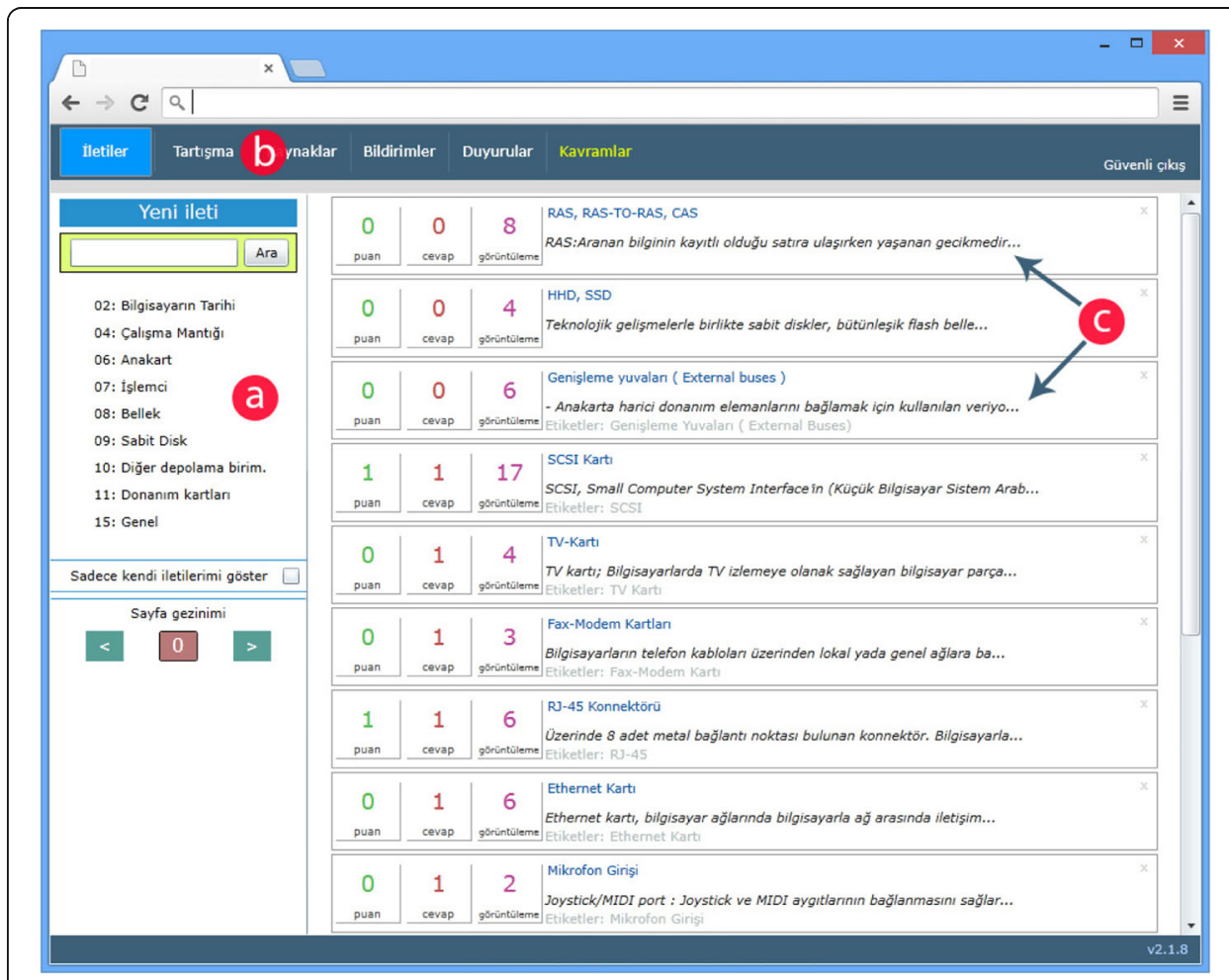

Fig. 1 Online Learning Environment 
system would be taken into consideration in their end-of-term evaluation, while no limits or obligations were stated regarding the activities to be conducted.

Data collected about the students' learning processes at the end of term consisted of 3803 sessions, 119,921 page visits, 4566 written texts, 62 questions in the discussion forum, 297 answers, 8601 evaluations, and 3937 tags used to label written messages. Students' interaction data in the online learning environment and their end-of term academic performance scores were used in the analysis.

\section{Data pre-processing and feature extraction}

Interactions carried out by students in the learning environment were taken into consideration in the determination of features that would be used in the prediction analyses and measurable metrics were established to reflect these interactions. The process of establishing features from raw data in the database was realized automatically via the help of an in-house developed tool. After the execution of the tool, a dataset is generated in which each row corresponds to a student and the columns represent the aggregated data regarding the extracted features. Features relating to the student interactions in the system were grouped under four headings: session, navigation, post and discussion. The list of extracted features and their descriptions can be reviewed in the Appendix: (Table 14). The selection of which of these features would be used in the analysis was decided in the pre-processing stage. Pre-processing carries notable importance in data mining studies. It is possible to generate better and easily understandable prediction models through interventions made at this stage.

The effect of two different pre-processing methods on the performance of classification algorithms were tested throughout the data analysis process. These included data transformation and feature selection techniques. The most frequently cited data transformation methods in the literature include the standardisation of data and the discretization of continuous data into categorical data (Ribeiro, Traina, \& Caetano Traina, 2008). This transformation could be carried out by the division of data into an equal number of units (equal frequency), division into intervals determined according to equal length cut-off points (equal width), or division in accordance with the cut-off points determined by the user (user-defined) (Dougherty, Kohavi, \& Sahami, 1995; Han, Kamber, \& Pei, 2006). The performance of classification models obtained through the selection of two different data transformation techniques were compared in this study. Firstly, the continuous features were divided into three equal units with the same frequency using the equal frequency method and then analysed. Secondly, the features were separated into three equal divisions of the same width using the equal width method and the same analysis was repeated with these features.

Another frequently used pre-processing technique in data mining studies is feature selection (Piramuthu, 2004). The aim of the feature selection is the determination of the more significant features in the prediction of target features in the prediction analyses. As such, it is possible to carry out the prediction analysis with a fewer number of features instead of all features. Various algorithms are used with the aim of feature selection and depending on the type of method 
used, these algorithms allocate a score for every feature. Subsequently, the features will be ranked according to this score and analyses are conducted with a selected number of features. In this study, feature selection was carried out with the help of the Rank widget in the Orange data mining tool (Demšar et al., 2013) using three different scoring techniques (e.g., Gain ratio, Gini index, and SVM weight) and the performance of obtained prediction models were compared. The rank widget scores the features based on selected scoring techniques and passes selected features to the prediction model.

\section{Academic performance}

The end-of-term grades of students in the Computer Hardware course were taken into consideration as an indicator of academic performance. The students sat three written exams in total; two mid-terms and one final examination. Both mid-term and final exams were in-house 45-min sitting exam, based on the lecture notes. Questions were formed at the knowledge, comprehension, and analysis levels. Exam papers were graded by two instructors. The final scores of students were calculated with a weighting of $25 \%$ for mid-terms along with $50 \%$ for the final examination. Descriptive statistics are given in Table 1. The academic performance used as a class feature within the scope of the study was converted into a categorical form taking into consideration the indicators defined in the university's undergraduate guidelines in the form of "Failed" $(n=27$, score $<=50)$ and "Passed" ( $n=49$, score $>50)$.

\section{Data analysis}

The establishment of a model that will predict students' end-of-term academic performance was treated as a binary classification problem. A high number of algorithms are present that could be used for classification purposes in data mining studies. The classification performance of algorithms can exhibit differences depending on the features of data set. As such, it is important to select an appropriate classification algorithm that suits the data set (C Romero, Espejo, Zafra, Romero, \& Ventura, 2013). When selecting the best performing classification algorithm based on performance measures, many researchers agree on repeating the same analysis via different algorithms to reach a working model (Akçapınar, Çoşgun, \& Altun, 2013; Osmanbegović \& Suljić, 2012; Cristobal Romero, Espejo, Zafra, Romero, \& Ventura, 2010). A similar approach was followed within the scope of this study. Seven different rule, tree, function and kernel-based algorithms frequently used in the literature for classification purposes were selected: Naive Bayes, Classification Tree, Random Forest, Support Vector Machines,

Table 1 Descriptive Statistics for Academic Performance

\begin{tabular}{llll}
\hline Exam & N & Mean & SD \\
\hline Midterm Exam 1 & 75 & 54.20 & 21.77 \\
Midterm Exam 2 & 73 & 52.33 & 17.92 \\
Final Exam & 74 & 60.59 & 17.51 \\
Final Score & 76 & 55.49 & 18.20 \\
\hline
\end{tabular}


Neural Network, CN2 Rules, and k Nearest Neighbours. The performance of classification algorithms was compared based on the performance metrics of Accuracy, Sensitivity, Specificity, and F-Measure. The obtained results were generalized using the 5-fold cross validation method, which splits the data into five folds and uses four folds for training and the remaining fold for testing. This procedure is repeated, so that each fold has been used for testing exactly once and performance metrics calculated based on the average performance of the model (Pretnar, 2019).

The classification analyses were carried out using the Orange data mining software (Demšar et al., 2013) in accordance with the process provided in Fig. 2. The whole set of interaction data was used within the scope of the first research question, whilst to answer the second reseach question, classification models were established by obtaining data belonging to weeks $3,6,9,12$, and 14 . When establishing classification models in order to develop an early warning system, the algorithm and the pre-processing methods that produced the best outcomes in the first research question were taken into consideration.

\section{Performance metrics}

The performance metrics to compare the performance of classification algorithms are calculated over and displayed together in a confusion matrix where instances classified correctly and incorrectly. A sample confusion matrix provided in Table 2 that belongs to a binary classification problem in the form of "Passed" and "Failed" regarding the academic performance of students was used in the explanation of performance metrics. Each row of the confusion matrix represents the instances of an actual class and each column represents the instances of a predicted class.

Here:

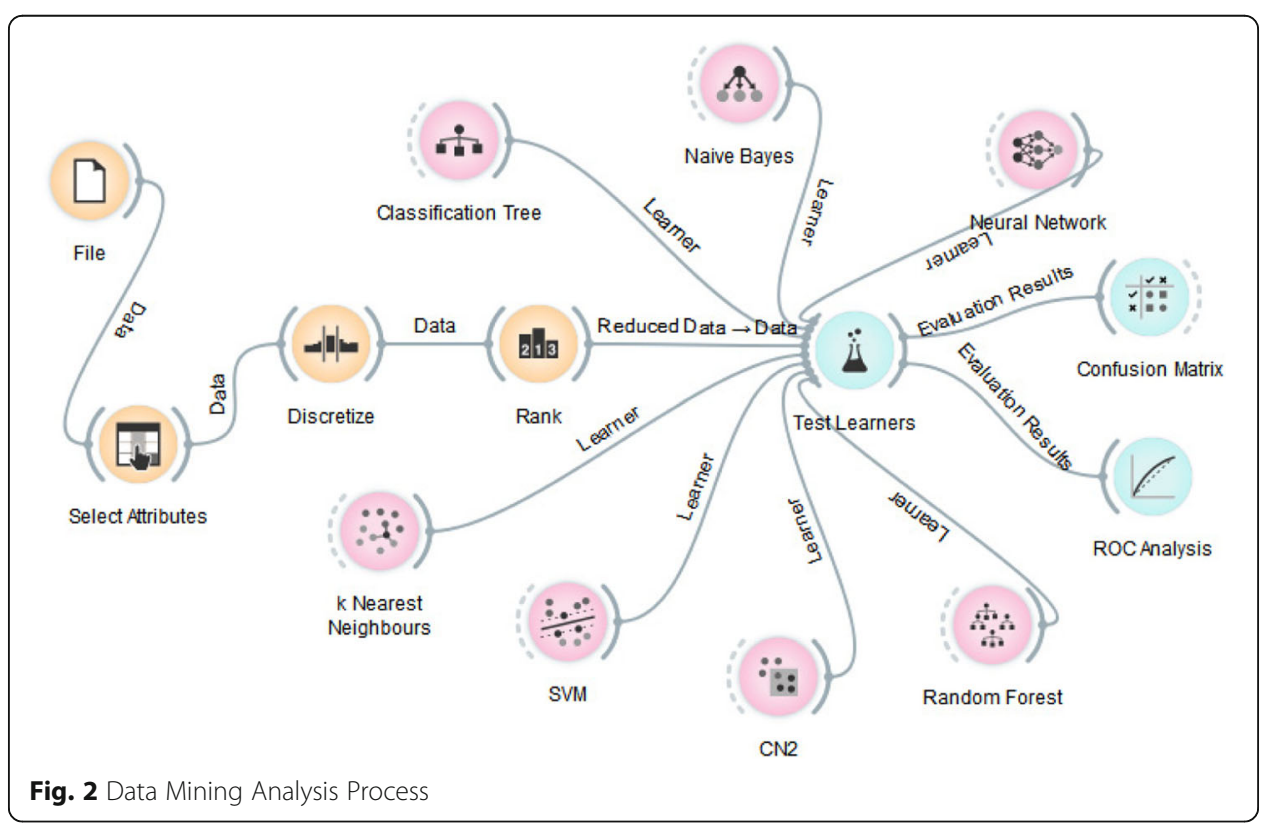


Table 2 Sample Confusion Matrix

\begin{tabular}{llll}
\hline & Predictions & & \\
\cline { 2 - 4 } & & Failed & Passed \\
\hline Actual Values & Failed & TN & FP \\
& Passed & FN & TP \\
\hline
\end{tabular}

TP (True Positive): The number of unsuccessful students that were classified as "Failed" by the model.

TN (True Negative): The number of successful students that were classified as "Passed" by the model.

FP (False Positive): The number of unsuccessful students that were classified as "Passed" by the model.

FN (False Negative): The number of successful students that were classified as "Failed" by the model.

The performance metrics according to this confusion matrix are calculated as below:

Accuracy: The ratio of subjects that are accurately classified by the model to the total number of subjects and is calculated by the below-provided formula. In cases where class features are not distributed in a well-balanced way, the obtained results could be misleading. As such, it is not a sufficient criterion on its own to measure the performance of the model.

$$
\text { Accuracy }=\frac{T P+T N}{T P+T N+F P+F N}
$$

Sensitivity: The probability of the model to classify "unsuccessful" students (Failed) correctly and is calculated according to the formula provided below. This metric is also called as Recall.

$$
\text { Sensitivity }=\frac{T P}{T P+F N}
$$

Specificity: The probability of the model to classify "successful" (Passed) students correctly and is calculated according to the formula provided below.

$$
\text { Specificity }=\frac{T N}{T N+F P}
$$

F-Measure: The use of sensitivity and specificity measures on their own could lead to the generation of biased comments in the evaluation of the models. The F-Measure,

\begin{tabular}{|c|c|c|c|c|c|}
\hline \multirow[t]{2}{*}{ No } & \multicolumn{2}{|l|}{ Pre-processing method } & \multirow[t]{2}{*}{ Classification algorithm } & \multirow{2}{*}{$\begin{array}{l}\text { Performance } \\
\text { metric }\end{array}$} & \multirow[t]{2}{*}{ Results } \\
\hline & Data Transformation & Feature Selection & & & \\
\hline 1 & None & None & \multirow{6}{*}{$\begin{array}{l}\text { Naive Bayes } \\
\text { Random Forest } \\
\text { Support Vector Machines } \\
\text { (SVM) } \\
\text { Classification Tree } \\
\text { Neural Network } \\
\text { CN2 Rules } \\
\text { kNN }\end{array}$} & \multirow{6}{*}{$\begin{array}{l}\text { Accuracy } \\
\text { Sensitivity } \\
\text { Specificity } \\
\text { F-Measure }\end{array}$} & Table 4 \\
\hline 2 & Equal frequency & None & & & Table 5 \\
\hline 3 & Equal width & None & & & Table 6 \\
\hline 4 & Equal width & Gain ratio & & & Table 7 \\
\hline 5 & Equal width & Gini index & & & Table 8 \\
\hline 6 & Equal width & SVM weights & & & Table 9 \\
\hline
\end{tabular}
which is the harmonic average of sensitivity and specificity, uses the two metrics together.

Table 3 Analyses Conducted within the Scope of the First Research Question 


$$
F-\text { Measure }=\frac{2 T P}{2 T P+F P+F N}
$$

\section{Results}

\section{Results regarding the first research question}

Six analyses were carried out with the aim of determining which pre-processing methods and algorithm would best predict student success using the whole data set (See Table 3, for details). In the first analysis, the classification performance was calculated without subjecting the data to any kind of operation (base model) while in the second and third analyses, the effect of different data transformation techniques on classification performance was tested. In the three subsequent analyses, the effects of different feature selection techniques on classification performance was investigated. It must be noted that the same classification algorithms, performance metrics, and 5-fold cross validation method was used in all analyses.

No changes were made to the features in the first analysis and all features were included in the analysis without undertaking any data transformation. The Neural Network algorithm reached the highest classification accuracy with a rate of $83 \%$. The other algorithms exhibited performance rates of $65 \%$ and above (Table 4).

The conducted analyses of results using the features established via the techniques of equal frequency and equal width are provided in Tables 5 and 6, respectively. When the performances of the data transformation methods were compared with the base model (Table 4), it was observed that the algorithms increased their classification performance in comparison to the based model in both data transformation methods (with the exception of SVM algorithm) (research question 1.1).

The use of the tree-based algorithms (Classification Tree and CN2 Rules) increased the classification accuracies by a range of $9 \%$ to $14 \%$ through the conversion of data into a categorical form. When comparing analyses conducted using the equal frequency and the equal width measure, the equal width technique exhibited a better performance in comparison to all algorithms in predicting students that failed (based on sensitivity metric).

Three different analyses were conducted to test which feature selection technique would produce the better outcome in the analysed data (research question 1.2). In the first analysis, the first ten features that received the highest scores according to the Gain ratio method were selected and performance metrics were calculated for all algorithms. In the second analysis, the first ten features that obtained the

Table 4 Data Transformation: None, Feature Selection: None

\begin{tabular}{lllll}
\hline Algorithm & Accuracy & Sensitivity & Specificity & F-Measure \\
\hline Classification Tree & 0.65 & 0.48 & 0.73 & 0.49 \\
CN2 Rules & 0.66 & 0.52 & 0.73 & 0.52 \\
Naive Bayes & 0.74 & 0.63 & 0.80 & 0.63 \\
Neural Network & 0.82 & 0.67 & 0.90 & 0.72 \\
kNN & 0.76 & 0.67 & 0.82 & 0.67 \\
Random Forest & 0.75 & 0.56 & 0.86 & 0.61 \\
SVM & 0.80 & 0.59 & 0.92 & 0.68 \\
\hline
\end{tabular}


Table 5 Data Transformation: Equal Frequency, Feature Selection: None

\begin{tabular}{lllll}
\hline Algorithm & Accuracy & Sensitivity & Specificity & F-Measure \\
\hline Classification Tree & 0.75 & 0.56 & 0.86 & 0.61 \\
CN2 Rules & 0.75 & 0.52 & 0.88 & 0.60 \\
Naive Bayes & 0.74 & 0.63 & 0.80 & 0.63 \\
Neural Network & 0.83 & 0.74 & 0.88 & 0.75 \\
kNN & 0.73 & 0.63 & 0.78 & 0.62 \\
Random Forest & 0.79 & 0.63 & 0.88 & 0.68 \\
SVM & 0.78 & 0.37 & 1.00 & 0.54 \\
\hline
\end{tabular}

highest scores were selected according to the Gini index and the classification analyses were repeated. Lastly, the ten features that received the highest score according to the SVM weight method were selected and the same analyses were repeated. The results of all three analyses are provided in Tables 7, 8, and 9. Features across all analyses were converted into categorical form according to the equal width method. The highest classification accuracy was reached by the CN2 Rules (86\%) and $\mathrm{kNN}(86 \%)$ algorithms and feature selection technique made in accordance to the Gini index (Table 9).

Classification results using 10 features selected according to the Gini index (Table 6) was more successful than the classification results using all of the features (Table 8) (research question 1.3). When the results are compared with respect to classification accuracies, the $\mathrm{kNN}$ and $\mathrm{CN} 2$ Rules algorithms increased their performance with a rate of $9 \%$. On the other hand, there was a fall in the correct classification rate of the Neural Network algorithm by $3 \%$ and it was observed that the correct classification rates of other algorithms did not change. On the other hand, with the exception of the decision tree, it was observed that all algorithms increased their predictive power in cases where a fewer number of features were used.

The best classification performance was obtained with features determined according to the Gini index. Upon examination of the features presented in Table 10, the following features were observed to be the most important in predicting academic performance: the logging of students on the system (total_session_count), logging on different days (unique_session_days), the time spent in the system (total_session_time), their visits (total_visits), writing down posts about the concepts they learned (total_posts_created), their tagging of written messages by using the concepts they learned (tag_used_count), the formation of

Table 6 Data Transformation: Equal Width, Feature Selection: None

\begin{tabular}{lllll}
\hline Algorithm & Accuracy & Sensitivity & Specificity & F-Measure \\
\hline Classification Tree & 0.79 & 0.81 & 0.78 & 0.73 \\
CN2 Rules & 0.77 & 0.59 & 0.86 & 0.64 \\
Naive Bayes & 0.75 & 0.63 & 0.82 & 0.64 \\
Neural Network & 0.83 & 0.78 & 0.86 & 0.76 \\
kNN & 0.77 & 0.67 & 0.82 & 0.67 \\
Random Forest & 0.78 & 0.67 & 0.84 & 0.68 \\
SVM & 0.75 & 0.44 & 0.92 & 0.56 \\
\hline
\end{tabular}


Table 7 Data Transformation: Equal Width, Feature Selection: Gain Ratio

\begin{tabular}{lllll}
\hline Algorithm & Accuracy & Sensitivity & Specificity & F-Measure \\
\hline Classification Tree & 0.76 & 0.78 & 0.76 & 0.70 \\
CN2 Rules & 0.80 & 0.81 & 0.80 & 0.75 \\
Naive Bayes & 0.80 & 0.81 & 0.80 & 0.75 \\
Neural Network & 0.82 & 0.74 & 0.86 & 0.74 \\
kNN & 0.83 & 0.78 & 0.86 & 0.76 \\
Random Forest & 0.78 & 0.67 & 0.84 & 0.68 \\
SVM & 0.78 & 0.59 & 0.88 & 0.65 \\
\hline
\end{tabular}

new tags that will be used in order to tag posts (tag_created_count), the writing of messages at regular intervals (unique_days_posted), the writing of answers to questions in the discussion forum (response_create_count), and the evaluation of written questions (question_rating_count) (research question 1.4).

It was concluded that the best classification rate was reached using the kNN and CN2 Rules algorithms along with the condition in which the equal width method and Gini feature selection technique was used (Table 8). The kNN algorithm displayed a better performance in predicting unsuccessful students in comparison to the $\mathrm{CN} 2$ algorithm and as such the progressive analyses were continued with the kNN algorithm. The confusion matrix for 5-fold cross validation derived via the $\mathrm{kNN}$ algorithm is provided in Table 11. The classification model correctly predicted 24 out of 27 (89\%) students that were failed at the end of term and 41 out of 49 (84\%) students that were passed. On the contrary, it classified 8 students $(16 \%)$ that were passed in reality as failed and classified 3 students (11\%) that were failed in reality as passed.

\section{Results regarding the second research question}

In order to investigate whether the students with a high probability of failure at the end of term could be identified in the earlier weeks, the data belonging to weeks $3,6,9$, and 12 were obtained from the database which contains the students' interaction data. The performance of the formed classification models was compared with the classification model established using the whole data (week 14). Ten features selected in accordance with the Gini index and kNN algorithm with the highest classification performance in the first research question was used in all established models. The data was converted into categorical form using the equal

Table 8 Data Transformation: Equal Width, Feature Selection: Gini Index

\begin{tabular}{lllll}
\hline Algorithm & Accuracy & Sensitivity & Specificity & F-Measure \\
\hline Classification Tree & 0.79 & 0.81 & 0.78 & 0.73 \\
CN2 Rules & 0.86 & 0.85 & 0.86 & 0.81 \\
Naive Bayes & 0.76 & 0.74 & 0.78 & 0.69 \\
Neural Network & 0.80 & 0.74 & 0.84 & 0.73 \\
kNN & 0.86 & 0.89 & 0.84 & 0.81 \\
Random Forest & 0.78 & 0.74 & 0.80 & 0.70 \\
SVM & 0.78 & 0.59 & 0.88 & 0.65 \\
\hline
\end{tabular}


Table 9 Data Transformation: Equal Width, Feature Selection: SVM Weights

\begin{tabular}{lllll}
\hline Algorithm & Accuracy & Sensitivity & Specificity & F-Measure \\
\hline Classification Tree & 0.79 & 0.81 & 0.78 & 0.73 \\
CN2 Rules & 0.83 & 0.74 & 0.88 & 0.75 \\
Naive Bayes & 0.80 & 0.74 & 0.84 & 0.73 \\
Neural Network & 0.82 & 0.70 & 0.88 & 0.73 \\
kNN & 0.83 & 0.74 & 0.88 & 0.75 \\
Random Forest & 0.76 & 0.70 & 0.80 & 0.68 \\
SVM & 0.79 & 0.56 & 0.92 & 0.65 \\
\hline
\end{tabular}

width method. The results regarding weeks $3,6,9,12$, and 14 with respect to the determined performance metrics are provided in Table 12.

According to the results presented in Table 12 and Fig. 3, the classification models established with the exception of the 6th week, displayed an increasing trend with respect to the classification accuracy.

The model established through the data obtained in the $14^{\text {th }}$ week classified $86 \%$ of students correctly, while the model established via the data obtained in the $3^{\text {rd }}$ week classified $74 \%$ of students correctly. In other weeks this ratio fluctuated between $71 \%$ and $84 \%$. Likewise, the value of the F-Measure, which is an indicator of the models to differentiate between successful and unsuccessful students, followed a rising trend with the exception of the $6^{\text {th }}$ week. In order to gain a greater understanding into how the obtained classification models perform on a weekly basis, confusion matrices regarding classification models are provided in Table 13.

When this set of data (provided below in visual form in Fig. 4) was analysed, it was observed that the classification models formed on a weekly basis correctly classified 20 out of 27 (74\%) unsuccessful students from week 3 onwards (TP value). In the last week, it classified 24 of them (89\%) correctly. However, the

Table 10 Important Features Determined According to the Gini Index and Explanations

\begin{tabular}{|c|c|c|c|}
\hline No & Feature & Gini index & Description \\
\hline 1 & question_rating_count & 0.083 & $\begin{array}{l}\text { The number of questions rated in } \\
\text { the discussion forum }\end{array}$ \\
\hline 2 & unique_session_days & 0.078 & $\begin{array}{l}\text { The number of different days on } \\
\text { which the learning log is recorded }\end{array}$ \\
\hline 3 & unique_days_posted & 0.076 & $\begin{array}{l}\text { The number of different days on } \\
\text { which posts are written }\end{array}$ \\
\hline 4 & total_posts_created & 0.056 & The total number of posts created \\
\hline 5 & total_visits & 0.042 & $\begin{array}{l}\text { The total number of visits made to } \\
\text { the learning materials }\end{array}$ \\
\hline 6 & total_session_count & 0.037 & The total number of sessions \\
\hline 7 & tag_used_count & 0.033 & $\begin{array}{l}\text { The number of tags used in the } \\
\text { written posts }\end{array}$ \\
\hline 8 & tag_created_count & 0.031 & $\begin{array}{l}\text { The number of new tags created } \\
\text { while writing post }\end{array}$ \\
\hline 9 & response_create_count & 0.028 & $\begin{array}{l}\text { The total number of responses } \\
\text { written in the discussion forum }\end{array}$ \\
\hline 10 & total_session_time & 0.024 & $\begin{array}{l}\text { The total amount of time (in minute) } \\
\text { spent in the learning environment }\end{array}$ \\
\hline
\end{tabular}


Table 11 Confusion Matrix for the kNN Algorithm*

\begin{tabular}{llll}
\hline & Failed & Passed & Total \\
\hline Failed & 24 & 3 & 27 \\
Passed & 8 & 41 & 49 \\
Total & 31 & 45 & 76 \\
\hline
\end{tabular}

*The columns represent the predictions and the rows represent the actual values

interpretation of this value on its own could lead to mistakes as the model can classify some successful students as unsuccessful by mistake (False Positive-FP). Upon examination of these values presented as FP in Table 13, it was observed that the classification model formed in the $3^{\text {rd }}$ week, classified 13 of the 49 (26\%) students that normally passed the subject as failed whilst this number dropped to $8(16 \%)$ in the last week.

\section{Discussion and conclusions}

The current study aimed to develop a model that enables the prediction of students' end-of-term academic performance earlier in the course using interaction data in an online learning setting. To that end, a two-stage analysis method was followed. In the first stage, the performance of the most widely cited classification algorithms in the literature were compared using the complete data set. At the same time, in the data pre-processing period, the impact of different techniques used for data transformation and feature selection known to have an effect on classification performance was tested. In the second stage, it was investigated whether the end-of-term performance of students could be predicted in earlier weeks using the selected algorithm, features, and data transformation techniques. At this stage, the performance of classification models formed with data obtained in weeks $3,6,9,12$, and 14 in predicting student academic performance was compared. Several performance metrics (e.g., Classification Accuracy, Sensitivity, Specificity, and F-Measure) were used in order to compare the performance of different classification models that were obtained. End-of-term scores in the Computer Hardware course were taken into consideration as an indicator of student academic performance, which was the target feature. Various features reflecting students' behaviour in the online learning setting were used to predict their end-of-term performance. The interaction of students in the learning setting was taken into consideration in the determination of these features. Academic performance was coded in the form of "Passed" or "Failed".

Table 12 Analysis Results Regarding Classification Models Established through Data Obtained in Different Weeks

\begin{tabular}{lllll}
\hline Week & Accuracy & Sensitivity & Specificity & F-Measure \\
\hline Week 3 & 0,74 & 0,74 & 0,73 & 0,67 \\
Week 6 & 0,71 & 0,81 & 0,65 & 0,67 \\
Week 9 & 0,76 & 0,74 & 0,78 & 0,69 \\
Week 12 & 0,84 & 0,81 & 0,86 & 0,79 \\
Week 14 & 0,86 & 0,89 & 0,84 & 0,81 \\
\hline
\end{tabular}




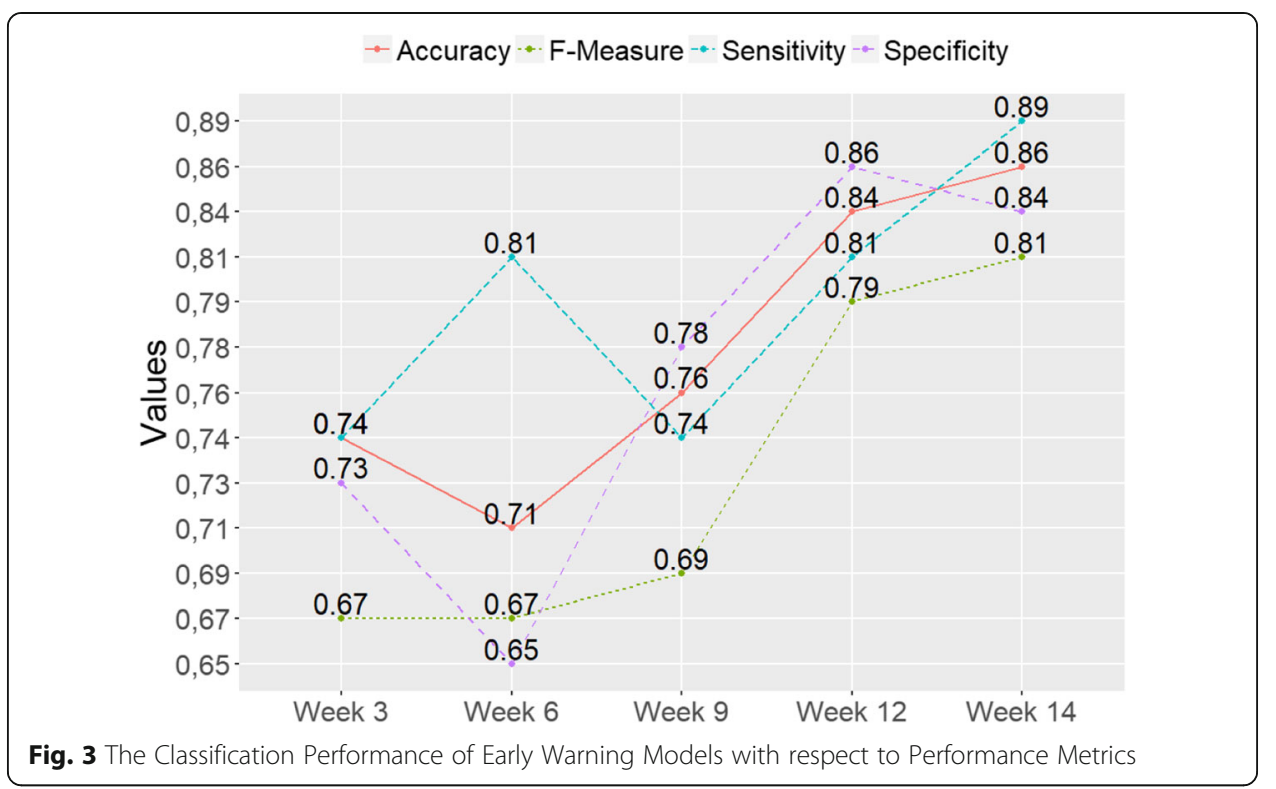

The effect of data transformation on classification performance was tested in the analysis stage. All features were converted into a categorical form using the techniques of equal frequency and equal width. Results indicated that models formed with categorical data performed better than models formed with continuous data. On the other hand, when different data transformation methods are compared, it was observed that the equal width technique produced better outcomes in comparison to the equal frequency method. The conducted studies indicate that categorical data give rise to better outcomes in comparison to continuous data in classification analyses (Cristobal Romero, Espejo, et al., 2013). At the same time, data converted into a categorical form may provide easier interpretation of obtained models by non-specialist individuals. Furthermore, it increases the reusability of the established models in different data sets, or, in other words, their generalizability.

Secondly, the impact of feature selection on classification performance was tested. For this purpose, the ten features which received the highest scores in accordance with the three different feature selection methods (Gain Ratio, Gini Index and SVM Weight) and the performance of the classification models using these features were compared with those that use all features (see Appendix). The results indicated that the classification performances obtained with 10 features selected according to the Gini index method was higher than the

Table 13 Confusion Matrices Regarding Classification Models Formed through Data Obtained in Different Weeks

\begin{tabular}{lllll}
\hline Week & TP & FP & TN & FN \\
\hline Week 3 & 20 & 13 & 36 & 7 \\
Week 6 & 22 & 17 & 32 & 5 \\
Week 9 & 11 & 38 & 7 \\
Week 12 & 20 & 7 & 42 & 5 \\
Week 14 & 22 & 8 & 41 & 3 \\
\hline
\end{tabular}




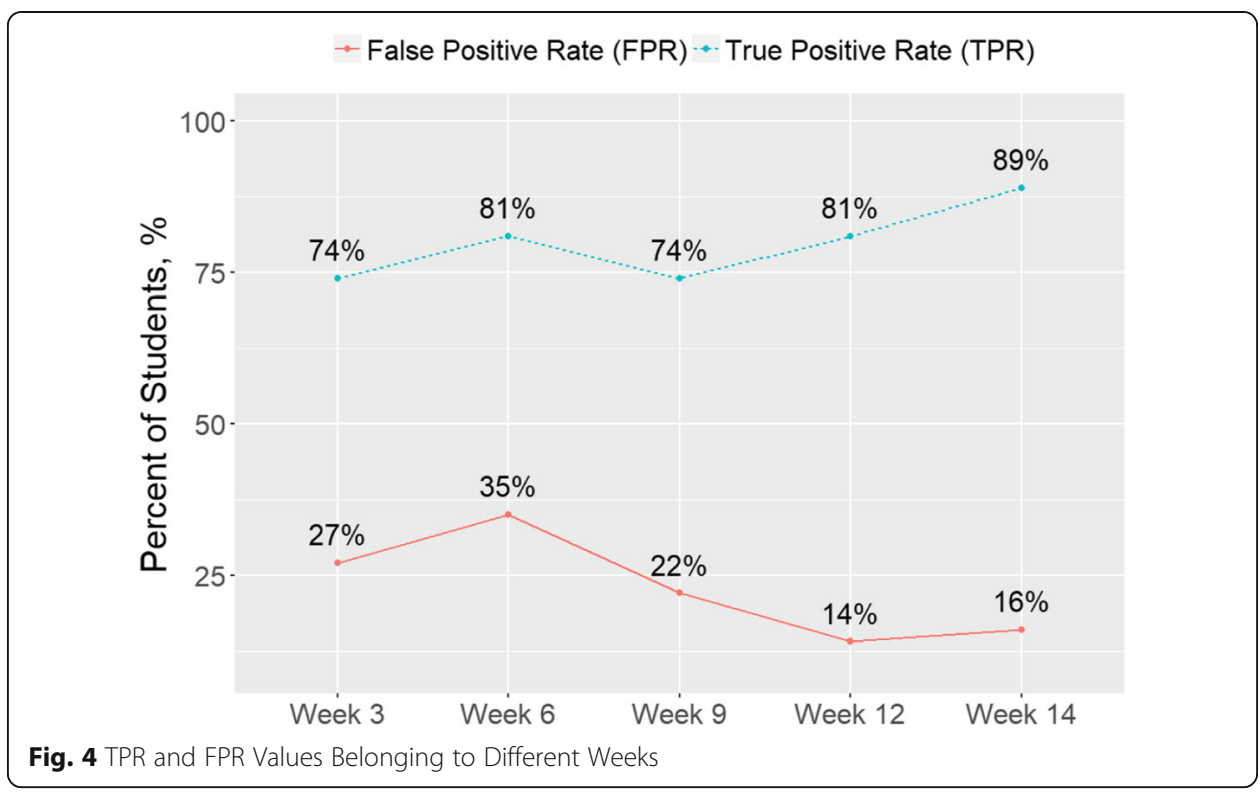

classification performances obtained through the use of all features. The feature selection method carries importance as it would enable the formation of prediction models through the use of a lower number of features. The use of a lower number of features means models that can be interpreted more easily.

Another intended purpose of the feature selection method is to gain an understanding of which features have the greatest impact on the structure (i.e. academic performance) that needs to be predicted (Baker, R. S. J. d., 2010). When the findings relating to important features were examined, it was observed that features such as regular logging of students onto the learning setting, the participation in the discussion forum, doing homework on a weekly basis, reading, and evaluating the content written by other students came to the fore. These findings are consistent with other studies. For example, in a study conducted in the Moodle setting, Lopez et al. (2012) reached the conclusion that the participation of students in the discussion forum is a good predictor of their success in lessons. Macfadyen and Dawson (2010) on the other hand established a regression model in order to predict the success of students in lessons using the interaction data in the Blackboard learning setting. It was found out that the most important feature in the model that would correctly predict a notable majority of students with the risk of failure was the number of posts sent to the discussion forum. The number of answers (response_create_count), which is an indicator of the participation of students in the discussion forum, along with the number of evaluations undertaken for the written questions (question_rating_count), became prominent as important features in the prediction of student performance.

Lavoué (2011) stated that the tagging feature used in online learning settings is important in the learning of new concepts, the formation of relationships in between concepts, and in supporting the cognitive and social learning processes of students to help them see the reflections of their internal concepts. The tagging of the written content (tag_used_count) and the addition of new tags to the setting (tag_created_count) emerge as important features in the prediction of student performance in this study as well. The Computer Hardware course in which the study was carried out is identical in 
that it is dominated by concepts and students are expected to learn 150-200 concepts throughout the semester. When examined from this aspect, the impact of features relating to tagging on academic performance was found to be important in encouraging the use of tagging in concept-dominated subjects. On the other hand, the regular logging-on of students in the setting and the time spent on it emerged as significant features. Akçapınar, Hasnine, Majumdar, Flanagan, and Ogata (2019), indicated the impact of logging onto learning settings regularly on academic success rather than carrying out many activities in one session.

In a comparison of the performances of classification algorithms developed using the complete data set, the highest level of classification accuracy was reached using the kNN and CN2 algorithms and under the condition in which data was converted into categorical form using the equal width method and important features selected according to the Gini index. Under these circumstances, the kNN and CN2 Rules algorithms classified $86 \%$ of students correctly while the other classification algorithms attained a classification accuracy of $76 \%$ and above. The classification model obtained with the $\mathrm{kNN}$ algorithm correctly predicted 24 of the 27 (89\%) failed students at the end of term, and 41 out of $49(84 \%)$ passed students. In contrast, it classified 8 students (16\%) that were passed in reality as failed and classified 3 students (11\%) that were failed in reality as passed.

In accordance with the findings obtained in the first stage, the classification models were formed with data that were obtained from weeks $3,6,9,12$, and 14 . The results of the analysis indicated that the model established in the $3^{\text {rd }}$ week correctly classified 20 out of 27 (74\%) failed students at the end of term but incorrectly classified 13 out of 49 (26\%) students who passed the subject as failed. Error rates gradually declined in models that were formed from data obtained in the progressive weeks. This result was expected as the data regarding student interactions in the system increases as the weeks progress. Costa et al. (2017) reported that the prediction performance of algorithms increased notably when students had completed $50 \%$ of the subjects.

One of the most important aims of educational data mining and learning analytics studies is to identify students with low performance and those experiencing problems and to provide the capability to change the behaviour underlying these conditions through timely interventions (Kimberly E. Arnold \& Pistilli, 2012). Feedback carries notable importance in the change of behaviour and the provision of instantaneous and individualised feedback to students is made possible through the developments in educational technologies (Bienkowski, Feng, \& Means, 2012; Tanes, Arnold, King, \& Remnet, 2011). The Signal project implemented by Purdue University indicated that students perceive individualised feedback given in an understandable format as positive (Bienkowski et al., 2012). The researchers used the information obtained through data mining analysis for the purpose of providing feedback to students in their study and showed that this was effective in increasing student success (K. E. Arnold, 2010). The prediction of unsuccessful students through data obtained in a short time frame such as the $3^{\text {rd }}$ week with a rate of $74 \%$ carries significance with respect to the prevention of possible failures. The mentioned data will gain teachers time to undertake pedagogical interventions (Costa et al., 2017) and will help lower the failure rates when shared with the students within the context of an effective feedback system. 


\section{Appendix}

Table 14 List of Extracted Features and their Descriptions

\begin{tabular}{|c|c|c|c|}
\hline No & Feature & Category & Description \\
\hline 1 & total_session_count & Session & The total number of sessions \\
\hline 2 & total_session_time & Session & $\begin{array}{l}\text { The total amount of time (in minute) spent in the learning } \\
\text { environment }\end{array}$ \\
\hline 3 & unique_session_days & Session & The number of different days on which the learning log is recorded \\
\hline 4 & total_visits & Navigation & The total number of visits made to the learning materials \\
\hline 5 & content_visit & Navigation & The total number of visits made to the content pages \\
\hline 6 & notification_visit & Navigation & The total number of visits made to the notification page \\
\hline 7 & announcement_visit & Navigation & The total number of visits made to the announcement page \\
\hline 8 & post_visit & Navigation & The total number of visits made to the post page \\
\hline 9 & discussion_visit & Navigation & The total number of visits made to the discussion page \\
\hline 10 & total_posts_created & Post & The total number of posts created \\
\hline 11 & post_writing_time & Post & The average amount of time (in second) spent for writing posts \\
\hline 12 & unique_days_posted & Post & The number of different days on which posts are written \\
\hline 13 & tag_created_count & Post & The number of new tags created while writing post \\
\hline 14 & tag_used_count & Post & The number of tags used in the written posts \\
\hline 15 & copy_paste_count & Post & The number of times using copy \& paste while writing posts \\
\hline 16 & keystroke_count & Post & $\begin{array}{l}\text { The average number of keystroke made by the student while writing } \\
\text { a post }\end{array}$ \\
\hline 17 & backspace_count & Post & $\begin{array}{l}\text { The average number of backspace key used by the student while } \\
\text { writing a post }\end{array}$ \\
\hline 18 & out-of-focus_count & Post & The average number of times the page lost focus while writing a post \\
\hline 19 & post_open_count & Post & The number of posts opened \\
\hline 20 & $\begin{array}{l}\text { comment_created_ } \\
\text { count }\end{array}$ & Post & The number of comments created \\
\hline 21 & post_rating_count & Post & The number of post rated \\
\hline 22 & $\begin{array}{l}\text { comment_rating_ } \\
\text { count }\end{array}$ & Post & The number of comments rated \\
\hline 23 & $\begin{array}{l}\text { response_create_ } \\
\text { count }\end{array}$ & Discussion & The total number of responses written in the discussion forum \\
\hline 24 & $\begin{array}{l}\text { response_open_ } \\
\text { count }\end{array}$ & Discussion & The total number of times to open responses in the discussion forum \\
\hline 25 & $\begin{array}{l}\text { response_rating_ } \\
\text { count }\end{array}$ & Discussion & The number of responses rated in the discussion forum \\
\hline 26 & $\begin{array}{l}\text { question_rating_ } \\
\text { count }\end{array}$ & Discussion & The number of questions rated in the discussion forum \\
\hline
\end{tabular}

\section{Abbreviations}

CART: Classification and Regression Tree; FN: False Negative; FP: False Positive; kNN: k-Nearest Neighbors; SVM: Support Vector Machines; TN: True Negative; TP: True Positive

\section{Acknowledgements}

Not applicable.

Authors' contributions

GA drafted the initial manuscript and conducted the research. AA provided insight and editing of the manuscript. PA provided supervision of the research. All authors read and approved the final manuscript.

\section{Funding}

Not applicable. 
Availability of data and materials

The datasets used and/or analyzed during the current study are available from the corresponding author on reasonable request.

\section{Competing interests}

The authors declare that they have no competing interests.

Received: 22 July 2019 Accepted: 2 October 2019

Published online: 31 October 2019

\section{References}

Akçapınar, G., Çoşgun, E., \& Altun, A. (2013, October 17th - 18th). Mining Wiki Usage Data for Predicting Final Grades of Students. Paper presented at the International Academic Conference on Education, Teaching and E-learning (IAC-ETeL 2013), Prague, Czech Republic.

Akçapınar, G., Hasnine, M. N., Majumdar, R., Flanagan, B., \& Ogata, H. (2019). Developing an early-warning system for spotting at-risk students by using eBook interaction logs. Smart Learning Environments, 6(4), 1-15. https://doi.org/ 10.1186/s40561-019-0083-4.

Arnold, K. E. (2010). Signals: Applying academic analytics. Educause Quarterly, 33(1) Retrieved from http://www.educause.edu/ ero/article/signals-applying-academic-analytics.

Arnold, K. E., \& Pistilli, M. D. (2012). Course signals at Purdue: Using learning analytics to increase student success. Paper presented at the proceedings of the 2nd international conference on learning analytics and knowledge, Vancouver, British Columbia, Canada.

Baker, R. S. J. d. (2010). Data Mining. In International encyclopedia of education (Third Edition) (pp. 112-118). Oxford: Elsevier.

Bienkowski, M., Feng, M., \& Means, B. (2012). Enhancing teaching and learning through educational data mining and learning analytics: An issue brief. Retrieved from Washington, D.C.:

Campbell, J. P., DeBlois, P. B., \& Oblinger, D. G. (2007). Academic analytics: A new tool for a new era. Educause Review, 42(4), 40

Chatti, M. A., Dyckhoff, A. L., Schroeder, U., \& Thüs, H. (2012). A reference model for learning analytics. International Journal of Technology Enhanced Learning, 4(5), 318-331. https://doi.org/10.1504/IJTEL.2012.051815.

Costa, E. B., Fonseca, B., Santana, M. A., de Araújo, F. F., \& Rego, J. (2017). Evaluating the effectiveness of educational data mining techniques for early prediction of students' academic failure in introductory programming courses. Computers in Human Behavior, 73(supplement C), 247-256. doi:https://doi.org/10.1016/j.chb.2017.01.047

Demšar, J., Curk, T., Erjavec, A., Gorup, Č., Hočevar, T., Milutinovič, M., ... Zupan, B. (2013). Orange: Data mining toolbox in python. Journal of Machine Learning Research, 14(1), 2349-2353.

Dougherty, J., Kohavi, R., \& Sahami, M. (1995). Supervised and unsupervised discretization of continuous features. Paper presented at the ICML.

Gašević, D., Dawson, S., Rogers, T., \& Gasevic, D. (2016). Learning analytics should not promote one size fits all: The effects of instructional conditions in predicting academic success. The Internet and Higher Education, 28, 68-84. https://doi.org/10. 1016/j.iheduc.2015.10.002.

Han, J., Kamber, M., \& Pei, J. (2006). Data Mining: Concepts and Techniques, Second Edition (The Morgan Kaufmann Series in Data Management Systems): Morgan Kaufmann.

Hu, Y.-H., Lo, C.-L., \& Shih, S.-P. (2014). Developing early warning systems to predict students' online learning performance. Computers in Human Behavior, 36, 469-478. https://doi.org/10.1016/j.chb.2014.04.002.

Johnson, L., Smith, R., Willis, H., Levine, A., \& Haywood, K. (2006). The 2011 horizon report. Austin, Texas: The new media consortium, 2011. In.

Lavoué, É. (2011). Social Tagging to Enhance Collaborative Learning. In H. Leung, E. Popescu, Y. Cao, R. H. Lau, \& W. Nejdl (Eds.), Advances in Web-Based Learning - ICWL 2011 (Vol. 7048, pp. 92-101): Springer Berlin Heidelberg.

Lopez, M. I., Luna, J. M., Romero, C., \& Ventura, S. (2012). Classification via clustering for predicting final marks based on student participation in forums. Paper presented at the 5 th international conference on educational data mining, EDM 2012, Chania, Greece.

Macfadyen, L. P., \& Dawson, S. (2010). Mining LMS data to develop an "early warning system" for educators: A proof of concept. Computers \& Education, 54(2), 588-599. https://doi.org/10.1016/j.compedu.2009.09.008.

Osmanbegović, E., \& Suljić, M. (2012). Data mining approach for predicting student performance. Economic Review, 10(1).

Peña-Ayala, A. (2014). Educational data mining: A survey and a data mining-based analysis of recent works. Expert Systems with Applications, 41(4, part 1), 1432-1462. doi:https://doi.org/10.1016/j.eswa.2013.08.042

Piramuthu, S. (2004). Evaluating feature selection methods for learning in data mining applications. European Journal of Operational Research, 156(2), 483-494. https://doi.org/10.1016/S0377-2217(02)00911-6.

Pretnar, A. (2019). The Mystery of Test \& Score. Retrieved from http://orange.biolab.si/blog/2019/1/28/the-mysteryof-test-and-score/

Ribeiro, M. X., Traina, A. J. M., \& Caetano Traina, J. (2008). A new algorithm for data discretization and feature selection. Fortaleza, Ceara, Brazil: Paper presented at the Proceedings of the 2008 ACM symposium on applied computing.

Romero, C., Espejo, P. G., Zafra, A., Romero, J. R., \& Ventura, S. (2010). Web usage mining for predicting final marks of students that use Moodle courses. Computer Applications in Engineering Education, n/a-n/a. https://doi.org/10.1002/ cae.20456.

Romero, C., Espejo, P. G., Zafra, A., Romero, J. R., \& Ventura, S. (2013). Web usage mining for predicting final marks of students that use Moodle courses. Computer Applications in Engineering Education, 21(1), 135-146. https:// doi.org/10.1002/cae.20456.

Romero, C., Olmo, J., \& Ventura, S. (2013). A meta-learning approach for recommending a subset of white-box classification algorithms for Moodle datasets. Paper presented at the 6th International Conference on Educational Data Mining (EDM 2013), Memphis, Tennessee, USA. 
Romero, C., Ventura, S., Hervás, C., \& Gonzales, P. (2008). Data mining algorithms to classify students. Paper presented at the Proc. Data Mining, Montreal: Int. Conf. Educ.

Tanes, Z., Arnold, K. E., King, A. S., \& Remnet, M. A. (2011). Using signals for appropriate feedback: Perceptions and practices. Computers \& Education, 57(4), 2414-2422. https://doi.org/10.1016/j.compedu.2011.05.016.

\section{Publisher's Note}

Springer Nature remains neutral with regard to jurisdictional claims in published maps and institutional affiliations.

Submit your manuscript to a SpringerOpen ${ }^{\odot}$ journal and benefit from:

- Convenient online submission

- Rigorous peer review

- Open access: articles freely available online

- High visibility within the field

- Retaining the copyright to your article

Submit your next manuscript at $\boldsymbol{\nabla}$ springeropen.com 